\title{
Multilinguales
}

\section{Processus de formation des expressions figées kabyles : cas de la polysémie}

Training process of fixed kabyle expressions : cases of polysemy

$$
\text { عملية تشكيل العبارات القبائلية الثابتة: حالات تعدد المعاني }
$$

\section{Mahdi Yahiaoui}

\section{Q OpenEdition}

\section{Journals}

Édition électronique

URL : http://journals.openedition.org/multilinguales/1150

DOI : 10.4000/multilinguales. 1150

ISSN : 2335-1853

Éditeur

Université Abderrahmane Mira - Bejaia

Référence électronique

Mahdi Yahiaoui, «Processus de formation des expressions figées kabyles : cas de la polysémie », Multilinguales [En ligne], 9 | 2018, mis en ligne le 01 juin 2018, consulté le 17 septembre 2019. URL: http://journals.openedition.org/multilinguales/1150; DOI : 10.4000/multilinguales.1150

Ce document a été généré automatiquement le 17 septembre 2019.

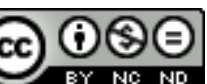

Multilinguales est mise à disposition selon les termes de la Licence Creative Commons Attribution Pas d'Utilisation Commerciale - Pas de Modification 4.0 International 


\title{
Processus de formation des expressions figées kabyles : cas de la polysémie
}

\author{
Training process of fixed kabyle expressions : cases of polysemy \\ عملية تشكيل العبارات القبائلية الثابتة:حالات تعدد المعاني
}

\section{Mahdi Yahiaoui}

1 Le figement est un phénomène propre et omniprésent dans toutes les langues naturelles. Il est

un processus inhérent aux langues naturelles par lequel des séquences linguistiques initialement employées comme séquences discursives libres se trouvent, pour des raisons diverses, particulièrement ou entièrement solidifiées (Mejri, 2000 : 609).

2 La langue kabyle compte dans son réservoir un nombre très important d'unités polysémiques qui contribuent de jour en jour à la formation de suites figées.

3 Cette contribution tente de retracer le processus de formation des unités phraséologiques kabyles. Nous entendons par processus l'ensemble des phénomènes qui contribuent à la formation des expressions figées, notamment le phénomène de la polysémie. En effet, nous exploitons les unités lexicales (verbes et noms) à tendances polysémiques pour tenter d'expliquer le rôle et l'importance des unités polysémiques dans la formation des expressions figées.

4 Il y a lieu de noter, tout d'abord, que le processus de figement en berbère demeure, à nos jours, le phénomène le plus énigmatique et le plus difficile à dépister. Juste en kabyle, le problème se pose sur plusieurs fronts :

5 D'abord, il existe plusieurs structures figées dont on ignore le processus de formation et, surtout, leur mode de fonctionnement.

6 Ensuite, les travaux qui sont censés recenser et décrire ces structures figées dans le domaine berbère sont peu nombreux et cela pose un sérieux problème au niveau théorique et méthodologique. 
7 Dans le domaine berbère, jusqu'à présent, les études qui ont été réalisées dans le cadre de la linguistique amazighe n'ont pas traité de la question de la formation des unités phraséologiques de manière approfondie. Autrement dit, à notre connaissance dans le domaine berbère, aucune étude n'a fait référence à la polysémie comme étant un facteur essentiel et pertinent dans la formation des unités phraséologiques.

L'idée que nous voulons développer dans cette contribution a émergé, à partir, d'une part, des constats que nous avons soulevés dans le cadre de notre travail de magister et d'autre part, des encadrements de quelques mémoires de licence et de master sur les expressions figées.

En effet, nous avons constaté la récurrence des unités polysémiques dans la plupart des expressions soumises à l'étude. Ce constat nous a laissé penser que la polysémie est un facteur pertinent qui joue un rôle primordial dans le processus de formation des suites figées en kabyle. En plus, nous avons aussi constaté que parmi les unités lexicales qui composent les expressions figées, il y a une unité de base qui est le noyau et c'est lui qui choisit une unité partenaire pour orienter et donner un sens à cette expression.

Pour démontrer le mode de formation de ces expressions, nous nous sommes basés sur les fondements de la sémantique interprétative de Rastier. Cette théorie se base sur l'interprétation du sens lexical pour aboutir à de grandes unités textuelles.

11 Les unités lexicales sont dotées de plusieurs sémèmes qui sont activés dans le discours selon les différentes situations d'énonciations.

12 Les expressions figées sont formées des unités polysémiques qui sont capables de renvoyer à plusieurs sens (dotés de plusieurs sémèmes).

13 Ces unités sont activées dans le discours et varient selon la situation de l'énonciation, il n'y a que le contexte qui peut déterminer le sens de l'unité ; «si le contexte comprend plusieurs types d'interprétants, plusieurs actualisations différentes, voire contradictoires, seront possibles pour définir le contenu d'un même signifiant, si bien qu'il pourra couvrir plusieurs sémèmes" (Rastier, 1987: 83-84). La fonction des unités polysémiques permet d'exprimer autant de choses (de réalités) par un simple mot.

14 Les expressions figées sont porteuses de deux sens, le sens transparent et le sens opaque. Elles sont donc comme des icebergs à deux faces; la face de surface ${ }^{1}$ qui est toujours explicable est interprétable (transparente); la face cachée ${ }^{2}$ qui est la moins accessible et la plus difficile à expliquer et à interpréter (opaque).

D'emblée, les unités les plus fréquentes dans le discours sont, en général, des unités à tendances polysémiques. Elles sont les plus favorites et les plus convoitées pour la formation des expressions phraséologiques. L'analyse qui suit repose principalement sur deux volets :

16 Le premier visera à recenser et à déterminer les sémèmes activés de chaque unité lexicale pour reconstituer le sens figé de l'expression.

17 Le deuxième sera consacré au rôle des unités lexicales à l'intérieur des expressions et aux types d'orientations qu'elles génèrent.

18 Le corpus que nous avons soumis à l'étude est essentiellement tiré du dictionnaire Kabyle-Français de Jean Marie Dallet. Nous n'avons choisi que quelques expressions figées, un choix qui est motivé par le fait que ces expressions sélectionnées sont formées à partir des unités polysémiques. 


\section{Le recensement des sémèmes et interprétation du} sens Les expressions figées sont formées ess
chaque unité regroupe plusieurs sémèmes.

\begin{tabular}{|l|l|l|l|}
\hline Unité 1 & Sémèmes de l'unité 1 & Sémèmes de l'unité 2 & Unité 2 \\
\hline \multirow{4}{*}{ Yečča } & Avaler & Tendresse & \\
\cline { 2 - 3 } & Attaquer & Amour & \\
\cline { 2 - 3 } & Recevoir & Affection & \\
\cline { 2 - 3 } & Perdre & Affection & \\
\cline { 2 - 3 } & Dissiper & Peur & \\
\cline { 2 - 3 } & Voler & Courage & \\
\cline { 2 - 3 } & Entrailles maternelles & \\
\hline
\end{tabular}

L'activation de l'un des sémèmes de chaque unité lexicale contribue à l'interprétation du sens figé de l'expression.

Ex.
(1). Yečča tasa-s
Il manger $(\mathrm{P})$ foie-son.
Il a mangé son foie.
Il ne se soucie de rien, il n'a aucun sentiment.

En observant cet exemple avec les traductions ${ }^{3}$ qui l'accompagnent, on se demande comment interpréter le sens de l'expression et comment on est arrivé à ce sens opaque?

Si on analyse d'un point de vue sémantique, chaque unité lexicale possède ses propres sémèmes, comme le montre le tableau ci-dessus.

En observant ce tableau, on peut constater que les unités lexicales sont dotées de plusieurs sémèmes que nous avons dépouillés dans le dictionnaire Kabyle-Français de Jean Marie Dallet. En effet, dans cet exemple, nous avons activé dans l'unité (yečča) les sémèmes (avaler, perdre), puis nous avons aussi activé dans l'unité (tasa-s) les sémèmes (tendresse, amour, affection et sentiment).

Il s'ensuit qu'une deuxième démarche est bien nécessaire pour faire le lien entre les différents sémèmes activés dans chaque unité lexicale afin de déduire le sens figé de l'expression.

Enfin, avec la combinaison des sémèmes, on obtient le sens de l'expression qu'on a dénommée comme opaque. combinaison, on obtient les sens suivants :

Avaler/sa tendresse 
Avaler son amour

Avaler son affection

Avaler ses sentiments

27 La deuxième combinaison est faite avec le sémème perdre.

Perdre sa tendresse

Perdre son amour

Perdre son affection

Perdre ses sentiments

Le sens figé (opaque) de cette expression est déduit de la combinaison des sémèmes activés dans chaque unité lexicale. Autrement dit, le sens figé d'une expression est une conséquence directe de l'association de deux unités polysémiques; chaque unité sélectionne un ou plusieurs sémèmes qui se combinent entre eux pour donner une lecture opaque à l'expression.

Parfois, il n'y a qu'un seul sémème qui est activé dans une unité lexicale, il s'agit des expressions qui ne sont dotées que d'un seul sens " des expressions monosémiques ».

(2). Yečča tidi-s

Il manger $(\mathrm{P})$ sueur-sa.

Il a mangé sa sueur.

Il lui a pris sa part (salaire, bien).

\begin{tabular}{|l|l|l|l|}
\hline Unité 1 & Sémèmes de l'unité 1 & Sémèmes de l'unité 2 & Unité 2 \\
\hline \multirow{4}{*}{ Yečča } & Avaler & Fatigue (après un travail rentable) & \\
\cline { 2 - 3 } & Attaquer & Peur & \\
\cline { 2 - 3 } & Recevoir & Chaleur & \multirow{2}{*}{ Tidi-s } \\
\cline { 2 - 3 } & Perdre & & \\
\cline { 2 - 3 } & Maladie & \\
\cline { 2 - 3 } & Dissiper & & \\
\cline { 2 - 4 } & Voler & & \\
\end{tabular}

Dans cet exemple, nous avons activé dans l'unité (yečča) le sémème (voler) et dans l'unité (tidi) le sémème (fatigue). En établissant le lien entre ces deux sémèmes, on obtiendra :

Voler sa fatigue

(3) Yečča lekwayed-is.

Il manger $(\mathrm{P})$ papiers-à lui.

Il a mangé ses papiers.

Il est fou, perdre raison. 




Le sens figé de l'expression s'explique par les connexions qui se font entre les différents
sémèmes de chaque unité lexicale. Comme nous l'avons déjà expliqué en haut, c'est au
contexte que revient le privilège d'établir la parfaite connexion entre les sémèmes
sélectionnés pour donner le sens figé de l'expression.

Le sens figé de l'expression s'explique par les connexions qui se font entre les différents
sémèmes de chaque unité lexicale. Comme nous l'avons déjà expliqué en haut, c'est au
contexte que revient le privilège d'établir la parfaite connexion entre les sémèmes
sélectionnés pour donner le sens figé de l'expression.

Le sens figé de l'expression s'explique par les connexions qui se font entre les différents
sémèmes de chaque unité lexicale. Comme nous l'avons déjà expliqué en haut, c'est au
contexte que revient le privilège d'établir la parfaite connexion entre les sémèmes
sélectionnés pour donner le sens figé de l'expression.

L'expression que nous avons présentée dans ce schéma ${ }^{4}$ est constituée de deux unités lexicales; (ečč /manger et lekwayed /papiers). Ainsi, dans (ečč /manger), nous avons sélectionné un seul sémème (sruh, dissiper/ perdre) et dans (lekwayed /papiers), c'est le sémème (nekwa, identité) qui est activé. On obtient comme résultat final de la combinaison des sémèmes choisis: perdre son identité

34 La lecture du sens des expressions figées n'est guère une tâche facile, derrière chaque sens réel se cache un sens métaphorique. Autrement dit, elles sont connues par leurs 
doubles sens ; le sens littéral et le sens spirituel. Une théorie inspirée de l'opposition entre le corps et l'âme (le premier sens voile le second et le second est le principal) (Rastier, 1987).

\section{Le rôle des unités de base dans les expressions}

Dans le domaine de la phraséologie, le processus de figement passe obligatoirement par quelques critères à savoir la polylexicalité, l'opacité sémantique et le blocage des propriétés paradigmatique qui sont indispensables pour la formation des expressions figées. Le choix des unités lexicales n'est guère un hasard, la sélection se fait d'une manière minutieuse et précise.

Sémantiquement parlant, une unité lexicale est susceptible de partager certaines affinités sémantiques avec un groupe d'unités et pas avec d'autres.

Ex.

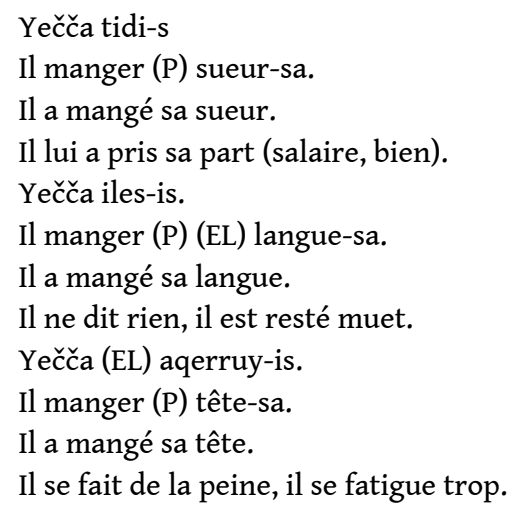

Dans le cas de ces expressions, le verbe "e ečč /manger " partage des liens sémantiques avec les autres éléments de l'expression, à savoir les noms tidi/ sueur, iles/ langue et aqerruy/ tête, les expressions sont toutes figées et le sens est toujours opaque. Toutefois, autant d'unités ne rentrent pas dans l'ordre de cette règle.

La question de figement est une question avant tout sémantique, si le sémème d'une unité lexicale n'est pas activé, le sens demeurera toujours libre et transparent.



39 Ces deux derniers exemples ne sont ni figés ni opaques, ils sont tout simplement des phrases simples. Les unités qui forment ces expressions n'ont pas perdu leur premier sens, même si les unités qui forment ces phrases sont polysémiques, on ne peut parler de figement pour la simple raison que le sens de ces unités est transparent.

Le comportement des unités lexicales présentes à l'intérieur de l'expression montre que toutes les expressions comptent une unité de base qui est le noyau fondamental de l'expression. Le noyau choisit un partenaire et oriente le sens de l'expression.

41 À l'intérieur des expressions figées, nous avons constaté l'existence d'une certaine hiérarchie. 
Entre éléments (affinités sémantiques) : dans A-B, A est souvent une base, qui détermine le fonctionnement syntaxique de l'UP, et $\mathrm{B}$ un collocatif sélectionné par la base (en fonction d'une intention discursive donnée); mais le pôle sémantique peut être aussi bien B, qui sélectionne A et le rapport devient presque un rapport d'implication réciproque (Pellen, 2001 : 614-615). (Positif)

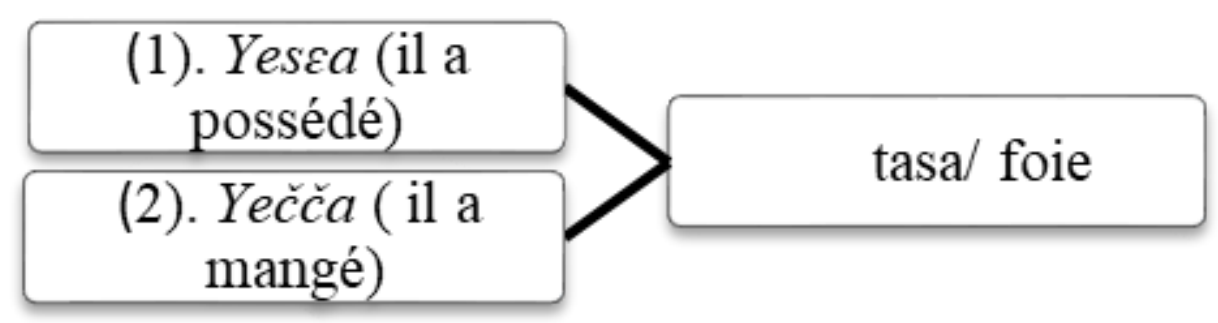

(Négatif) 

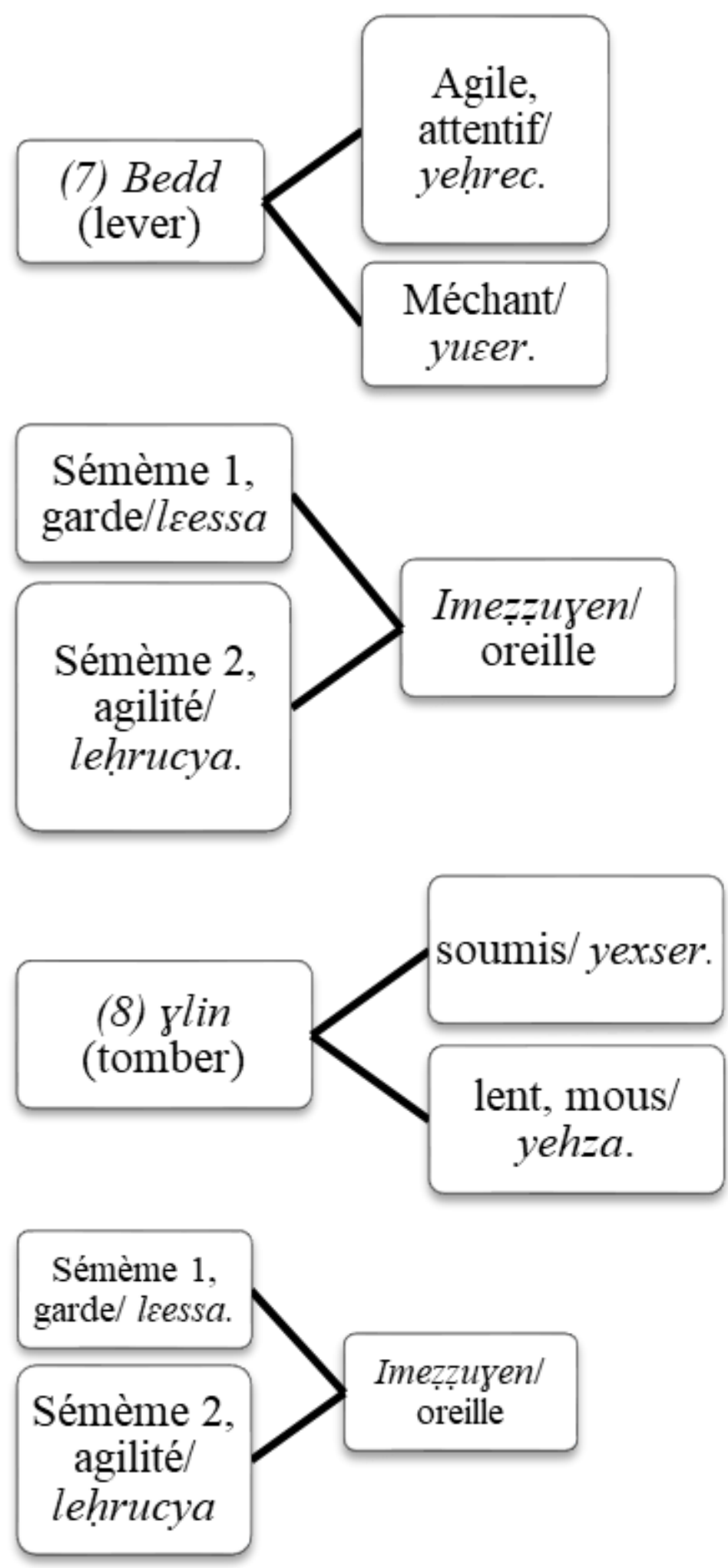

Bas / faible

En prêtant attention à ces deux exemples, on peut en constater que le sens est graduel, il est orienté du bas vers le haut en affectant directement le sens de l'expression. Nous avons gardé les mêmes sémèmes de l'unité de base Imez̧zưen/ oreille, que ce soit dans l'exemple (7) ou (8) et nous avons conclu que c'est uniquement la fréquence sémantique 
des deux expressions qui changent. Sens à fréquence forte, exemple (7) et sens à fréquence faible pour l'exemple (8).

Ici et là-bas/ ailleurs

L'orientation du procès joue un rôle très important dans le fonctionnement des expressions figées. Le rapport sémantique change de camp par rapport au locuteur, ce rapport est exprimé par la particule d'orientation spatiale ( $d$ - /vers ici).

(9). Yefka afus

Il donné $(\mathrm{P})$ main

Il a donné la main

Trahir, dénoncer.

(10). Yefka-d afus

Il donner (P)- vers ici main (EL)

Il a donné la main

Aider

51 L'unité de base dans les deux expressions est bel et bien afus / main, le sémème activé dans les deux expressions est lié à la force. En comparant l'expression (9) et (10) sur le plan formel, il n'y a qu'un seul élément qui diffère dans l'exemple (10), c'est la particule d'orientation spatiale. Par conséquent, le rapport de force change de position et du coup influe sur le sens global de l'expression.

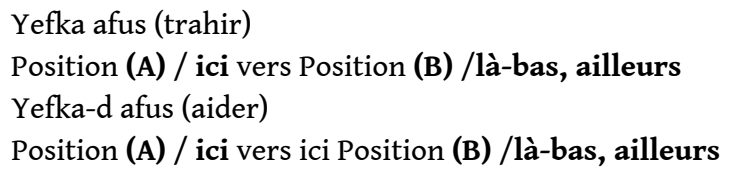

Donner la main à un ennemi, c'est augmenter ses forces et ses capacités de vaincre. Quitter un camp pour rejoindre un autre camp ennemi est considéré comme un acte de trahison.

Par ailleurs, le sens inverse orienté par la particule d'orientation spatiale (-d/ vers ici), de la position (B) (là-bas, ailleurs) vers la position (A) (ici) est noble et digne. En effet, il n'y a pas que le rapport de force qui change dans les expressions figées, mais il faut puiser dans le fond des expressions afin de trouver d'autres rapports.

Les unités lexicales qui contribuent à la formation des expressions figées sont des unités purement polysémiques. Le sens global de l'expression est le résultat de la fusion d'un sémème activé dans l'unité de base avec un autre activé dans l'unité partenaire. Cette dernière oriente le sens global de l'expression vers le positif /négatif, haut/ bas, ici/ là-bas, etc.

Enfin, le domaine de la phraséologie kabyle reste l'un des domaines les plus passionnants à étudier, autant de problématiques et de pistes restent à explorer, les portes sont ouvertes sur les différentes théories linguistiques et ce dans l'objectif de porter des éclaircissements sur la question de formation des suites figées, autrement dit sur le processus de figement.

56 Notre travail consiste à démontrer le processus de formation des expressions figées kabyles. Il est basé uniquement sur les unités polysémiques dont l'objectif est de démontrer le rôle des sémèmes dans la détermination du sens figé et celui des unités lexicales dans l'orientation du sens.

57 Après analyse des expressions, nous avons conclu :

Le sens figé d'une expression est toujours le résultat d'une combinaison entre les sémèmes des unités lexicales. 
En testant les unités lexicales à l'intérieur des expressions, nous avons dégagé deux types d'unités ; la première est la base ; la seconde est l'unité partenaire.

C'est l'unité de base qui oriente le sens de l'expression. En nous basant sur les exemples que nous avons analysés, nous avons dégagé trois types d'orientation: Le positif ou le négatif, le haut et le bas, ici et là-bas/ ailleurs.

\section{BIBLIOGRAPHIE}

DALLET, J. M., Dictionnaire kabyle- Français, parler des Ait Menguellat, éditions SELAF, Paris, 1982.

GROSS, G., Les expressions figées en français (noms composés et autres locutions), édition OPHRS, 1996.

REY, I. G., La phraséologie du français, édition Presse Universitaire du Midi, 1996.

HEBERT, L., « La sémantique interprétative », dans Louis Hébert (dir.), Signo [en ligne], Rimouski (Québec), : disponible sur [http://www.signosemio.com/rastier/semantique-interpretative.asp]. MEJRI, S., « le figement lexical », : disponible sur [www.lli.univparis13.fr/membres/biblio/ 1354_fige_lex.doc].

PELLEN, R., « Phraséologie et phraséographie en espagnol. De la typologie à l'inventaire des ressources ", In Bulletin Hispanique, tome 103, n² 2, pp. 607-674, 2001. doi : 10.3406/hispa.5090 PICOCHE, J., Précis de lexicologie française, l'étude de l'enseignement du vocabulaire, édition NATHAN UNIVERSITE, 1997.

RASTIER F., Sémantique interprétative, Presse Universitaire de France, Paris, 1987.

REY, A, Chanterreau, Dictionnaire d'expressions et locutions, Le Robert, imprimé en Italie par la tipographica Verese Srl, 2015.

SVENSSON, M. H., Critères de figement identification des expressions figées en français contemporain, Umea Universitet, 2004.

TILIKETE, O., Les locutions à noyau verbal en Kabylie, approche syntaxique, sémantique, pragmatique et rhétorique, tome 1, INALCO Paris, 1999.

VIELLARD, S., « Quelques remarques sur phraséologie historique du russe », in Stéréotypie et figement à l'origine du sens, sous la direction de Vladimir Beliakov et Salah Mejri (dir.), édition Presses universitaires du Midi, 2015.

YAHIAOUI, M., Essai de typologie syntaxique des expressions figées kabyles (parlers de Tichy), mémoire de magister, université Abderrahmane Mira, Bejaia, 2009.

\section{NOTES}

1. La face de surface regroupe le premier sens de l'unité lexicale, les sens qui sont connus par les interlocuteurs qui parlent une même langue. Ex. Ečč (manger) : est l'action de prendre de la nourriture et de l'avaler. C'est en quelque sorte le premier sens qui saute aux yeux. 
2. La face cachée concerne les sens qu'une unité linguistique peut regrouper, c'est une face difficile à cerner car plusieurs facteurs rentrent dans l'interprétation sémantique de l'unité en question.

3. Les expressions figées sont suivies de plusieurs traductions :

Traduction littérale

Traduction intégrale (équivalent de l'expression en français)

Le sens figé de l'expression.

4. Dans cet exemple, il y a changement de schéma, c'est juste pour mieux voir l'intersélection des sémèmes de chaque unité lexicale.

5. Selon la sémantique interprétative, quatre composantes structurent le plan sémantique des textes (le plan du contenu, des signifiés, par opposition à celui de l'expression, des signifiants) : la thématique (les contenus investis), la dialectique (les états et processus et les acteurs qu'ils impliquent), la dialogique (les évaluations modales, par exemple véridictoires: le vrai/faux, thymiques: le positif/négatif) et la tactique (les positions linéaires des contenus) Louis Hébert (2006). La référence ne figure pas en bibliographie.

\section{RÉSUMÉS}

Cette étude vise à démontrer le processus de formation des expressions figées kabyles. Ce travail est basé essentiellement sur le rôle des unités polysémiques dans la formation des expressions figées. La première partie est donc consacrée au recensement des sémèmes pour reconstituer le processus de formation du sens figé. La seconde partie est consacrée à déterminer le rôle des unités polysémiques et comment elles orientent le sens à l'intérieur des expressions.

This study aims to demonstrate the process of formation of static Kabyle expressions. This work is essentially based on the role of polysemic units in the formation of fixed expressions. The first part is devoted to the census of the sememes to reconstitute the process of formation of the fixed sense. The second part is devoted to determining the role of polysemic units and how they orient meaning within expressions.

\section{INDEX}

Mots-clés : phraséologie, figement, expression, polysémie

Keywords : phraséology, congestion, expression, polysemy

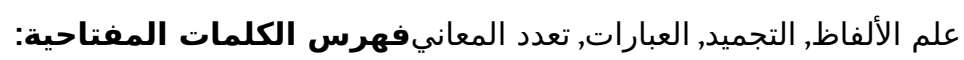

\section{AUTEUR}

\section{MAHDI YAHIAOUI}

Université de Bejaia 